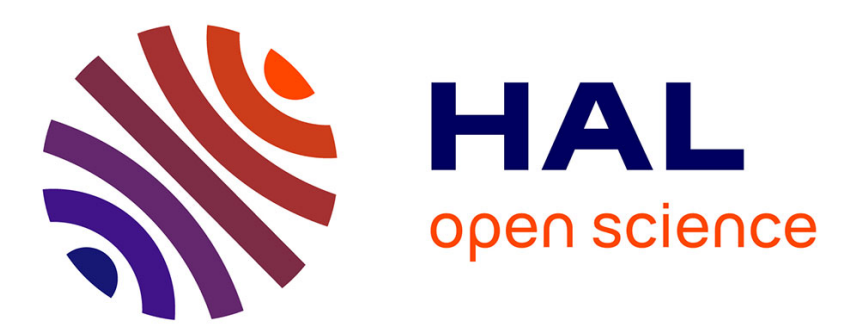

\title{
Observability of structured linear systems in descriptor form: a graph-theoretic approach
}

Taha Boukhobza, Frédéric Hamelin, Dominique Sauter

\section{To cite this version:}

Taha Boukhobza, Frédéric Hamelin, Dominique Sauter. Observability of structured linear systems in descriptor form: a graph-theoretic approach. Automatica, 2006, 42 (4), pp.629-635. 10.1016/j.automatica.2005.12.001 . hal-00120927

\section{HAL Id: hal-00120927 https://hal.science/hal-00120927}

Submitted on 18 Dec 2006

HAL is a multi-disciplinary open access archive for the deposit and dissemination of scientific research documents, whether they are published or not. The documents may come from teaching and research institutions in France or abroad, or from public or private research centers.
L'archive ouverte pluridisciplinaire HAL, est destinée au dépôt et à la diffusion de documents scientifiques de niveau recherche, publiés ou non, émanant des établissements d'enseignement et de recherche français ou étrangers, des laboratoires publics ou privés. 


\title{
Observability of structured linear systems in descriptor form: a graph-theoretic approach
}

\author{
T. Boukhobza, F. Hamelin and D. Sauter \\ Henri Poincaré University Nancy 1, CRAN UMR 7039 CNRS-INPL-UHP, BP 239, 54506 Vandæuvre Cedex, France. \\ Phone: 33383684 464, Fax: 33383684 462, email: taha.boukhobza@cran.uhp-nancy.fr
}

\begin{abstract}
This paper deals with the right-hand side observability of structured linear systems in descriptor form. The method we propose is based on a graph-theoretic approach. It is applicable to a large class of descriptor systems including regular and nonregular systems even if they are square, underdetermined or overdetermined. After defining digraphs which represent such systems, we express in graphic terms necessary and sufficient conditions to achieve the observability of the whole or of a given part of the state. These conditions are easy to check because they are based on comparison of integers and on finding edges and subgraphs in a digraph. Therefore, our approach is suited to study large scale and/or uncertain systems.
\end{abstract}

Key words: Descriptor systems, observability, structured systems, graph theory.

\section{Introduction}

During the last decades, a large amount of attention has been paid to the graphical analysis of dynamical system properties. This approach is mainly dedicated to linear systems in standard form for which many structural properties such as controllability, observability, solvability of several classical control problems including disturbance rejection, inputoutput decoupling, fault detection and isolation are studied. Survey paper (Dion et al., 2003) reviews the most significant results in this area. Unfortunately, not so many papers have dealt with the graphical analysis of the properties of descriptor systems. Effectively, descriptor systems are more complicated to study than linear systems in standard form. Descriptor systems result from a convenient and natural process modeling (Lewis, 1992) and their applications can be found in various fields (Müller, 2000) such as robotics, electrical circuit networks, biologic and economic systems. Moreover, considering a system with unknown inputs is quite easy using descriptor forms since unknown inputs, like faults or disturbances, can be included in an extended state of descriptor system, which may then be non-square and non-regular. All these features increase interest of such systems.

Structural properties like solvability, controllability and observability are revisited for descriptor systems in (Aplevich, 1991; Cobb, 1984; Dai, 1989; Özccaldiran et al., 1992; Yip and Sincovec, 1981). Among these properties, the observability analysis of the whole or of a given part of the state for descriptor systems is a great interest mainly in the design of control laws, fault detection and isolation schemes, fault tolerant control strategies, supervision proposals and so on. The observability characterization is also an important stage in the design of state or unknown input observers for descriptor systems. Indeed, most existence conditions of observers devoted to descriptor systems (Hou and Müller, 1999b; Nikoukah et al., 1998; Darouach et al., 1996; Chu and Mehrmann, 1999) are related to some sufficient observability conditions.

Until now, in most cases, the studies concerning the observability of descriptor systems deal with algebraic and geometric tools. Yet, the use of such tools to analyse the observability of descriptor systems assumes the exact knowledge of the state space matrices which characterize the model of the system. In many modeling problems, these matrices have a number of fixed zero entries, determined by the physical structure, while the remaining entries are not exactly known. To study the properties of these systems in spite of the poor knowledge we have of them, the idea is that we only keep the zero/nonzero entries in the matrices of the state-space representation. Thus, we consider models where the fixed zeros are conserved while the nonzero entries are replaced by free parameters. There is a huge amount of interesting studies in the literature using this kind of models called structured models. These models capture most of the available structural information from physical laws. Moreover, they are helpful to handle uncertain systems i.e. systems where parameters values are not exactly known. Finally, their study requires a low computational burden which allows us to deal with large scale systems. Because of these characteristics, we think that structured systems are particularly adapted to 
study the observability. Subsequently, in this paper, we consider only structured linear systems in descriptor form (or structured descriptor systems):

$$
\Sigma_{\Lambda}:\left\{\begin{array}{c}
E^{\lambda} \dot{x}=A^{\lambda} x+B^{\lambda} u \\
y=C^{\lambda} x \\
z \stackrel{\text { def }}{=} E^{\lambda} x
\end{array}\right.
$$

where $x \in \mathbb{R}^{n}, \quad u \in \mathbb{R}^{m}$ and $y \in \mathbb{R}^{p}$ are respectively the state vector, the input vector and the output vector. $E^{\lambda} \in \mathbb{R}^{q \times n}, A^{\lambda} \in \mathbb{R}^{q \times n}, B^{\lambda} \in \mathbb{R}^{q \times m}$, and $C^{\lambda} \in \mathbb{R}^{p \times n}$ represent matrices which elements are either fixed to zero or assumed to be nonzero free parameters noted $\lambda_{i}$. The set of these parameters is $\Lambda=\left\{\lambda_{1}, \lambda_{2}, \ldots, \lambda_{h}\right\}$. New variable $z \in \mathbb{R}^{q}$ called dynamic variable, is introduced in this paper.

If all the nonzero parameters $\lambda_{i}$ are fixed, we obtain an admissible realization of structured system $\Sigma_{\Lambda}$. The properties of each realization can be studied according to the values of $\lambda_{i}$. We say that this property is generically true if it is true for almost all the realizations of the system.

Among the studies analysing structured systems in descriptor form by means of graph-theoretic approach, we can cite (Reinschke and Wiedemann, 1997; Wiedemann, 1999). In (Reinschke and Wiedemann, 1997), the authors use digraphs to characterize structural controllability of descriptor system. However, they make some assumptions about the studied systems: matrices $E^{\lambda}$ and $A^{\lambda}$ have to be square $(q=n), \operatorname{det}\left[s E^{\lambda}-A^{\lambda}\right] \not \equiv c s t$ and no main diagonal element of $\left[s E^{\lambda}-A^{\lambda}\right]$ equals zero. Many descriptor systems do not satisfy all these assumptions like the underdetermined ones which are very helpful to represent systems with unknown inputs. In (Wiedemann, 1999), structural properties of regular descriptor systems ${ }^{1}$, including the observability, are studied by assuming also that there is no zero element in the main diagonal of $\left[s E^{\lambda}-A^{\lambda}\right]$. In particular, using a graphtheoretic approach, necessary and sufficient conditions for $R$-observability (which is equivalent to the right-hand side observability (Hou and Müller, 1999a) property for regular descriptor systems ) and impulse-observability are given.

The aim of this paper is to study the right-hand side observability (Hou and Müller, 1999a) which do not necessarily satisfy the previous assumptions. Indeed, the regularity assumption on descriptor systems as well as the hypothesis on the main diagonal of $\left[s E^{\lambda}-A^{\lambda}\right]$ are dropped through this paper. That means that our method is applicable also to nonregular and/or non-square descriptor systems.

The paper is organized as follows: after section 2 , which is devoted to the problem formulation, a digraph representation of structured systems is given in section 3. Necessary and sufficient conditions for the right-hand side observability of the whole or any given part of the state of the system are provided in section 4 . The proposed approach is illustrated with an example in section 5. Finally, a conclusion ends the paper.

\footnotetext{
1 System $\left(\Sigma_{\Lambda}\right)$ is generically regular when matrices $E^{\lambda}$ and $A^{\lambda}$ are square and when for almost all its realizations, $\operatorname{det}\left[s E^{\lambda}-A^{\lambda}\right] \not \equiv 0$.
}

\section{Problem statement}

First of all, let us precise hereafter the assumptions made on the system and the type of observability we study.

Obviously, in order to guarantee that there exists at least one trajectory $x(t)$ satisfying relations $(1), u(t)$ and $\operatorname{Ex}\left(0_{-}\right)$ are assumed to be admissible i.e. they do not result in contrary equations in $\left(\Sigma_{\Lambda}\right)$ and are such that system (1) is solvable. This assumption is quite natural and readers interested by the solvability of descriptor systems can refer to (Geerts, 1993; Yip and Sincovec, 1981). Moreover, in addition to the previous hypothesis and without loss of generality, we only assume that $E^{\lambda}$ does not have any row identically zero. However, this assumption is not restrictive.

We consider, in this paper, underdetermined, overdetermined or square structured descriptor systems. Moreover, no assumption is done on the rank of $\left[s E^{\lambda}-A^{\lambda}\right]$ neither on its main diagonal. Therefore, our approach is adapted to analyse the input observability (Hou and Patton, 1998) of systems with unknown inputs since we do not made any hypothesis on the regularity of system $\left(\Sigma_{\Lambda}\right)$. The kind of observability we study is related to the right-hand side (r-h.s.) observability (Hou and Müller, 1999a), which is equivalent to the $R$-observability (Dai, 1989; Yip and Sincovec, 1981) or to the finite observability (Verghese et al., 1981) for regular descriptor systems. Hereafter, we recall the definition of the r-h.s. observability. This definition extends the one given in (Hou and Müller, 1999a) for non-structured descriptor systems:

Definition 1 Structured system $\left(\Sigma_{\Lambda}\right)$ is generically $r$-h.s. observable, if, for almost all the realizations of structured system $\left(\Sigma_{\Lambda}\right), y(t)=0$ for $t \geq 0$ implies $x\left(0_{+}\right)=0$.

The property of r-h.s. observability is a very important observability property for descriptor systems. Indeed, it is proved in (Hou and Müller, 1999b), that it is closely related to the finite detectability which ensures the existence of a generalized observer which allows to reconstruct the state. In fact, $\left(\Sigma_{\Lambda}\right)$ is r-h.s. observable iff for $t>0$, state $x(t)$ can be uniquely determined using the knowledge of the output and the input (Müller and Hou, 1993; Hou and Müller, 1999b) and so equivalently iff there exist generically integers $k$ and $l$ and structured matrices $G_{y}^{\lambda}$ and $G_{u}^{\lambda}$ such that $x(t)=G_{y}^{\lambda}\left(y(t)^{T}, \dot{y}(t)^{T}, \ldots, y^{(k)}(t)^{T}\right)^{T}+$ $G_{u}^{\lambda}\left(u(t)^{T}, \dot{u}(t)^{T}, \ldots u^{(l)}(t)^{T}\right)^{T}, \forall t>0$.

To summarize, considering structured system in descriptor form $\left(\Sigma_{\Lambda}\right)$, we propose to answer the question whether or not the whole or any given part of state $x(t)$ is r-h.s. observable. It turns out that this question can be answered by checking some simple graphic conditions.

\section{Digraph representation of a structured descriptor system}

This section is devoted to the definition of a digraph used to represent structured descriptor system $\left(\Sigma_{\Lambda}\right)$. This digraph is noted $\mathcal{G}\left(\Sigma_{\Lambda}\right)$ and is constituted by a vertex set $\mathcal{V}$ and an edge set $\mathcal{E}$. The vertices are associated to variables $x(t), y(t)$ and 
$z(t)$ of $\left(\Sigma_{\Lambda}\right)$ and the directed edges represent links between these variables. For the sake of simplicity, known input $u(t)$ is not taken into account in $\mathcal{G}\left(\Sigma_{\Lambda}\right)$ since it is well known that the r-h.s. observability of descriptor systems depends only on matrices $E^{\lambda}, A^{\lambda}$ and $C^{\lambda}$ (Hou and Müller, 1999a). The edge direction is adapted to the observation problem since it corresponds to the knowledge-flow used to observe the state from the outputs and their derivatives. More precisely, $\mathcal{V}=\mathbf{X} \cup \mathbf{Y} \cup \mathbf{Z}$ where $\mathbf{X}=\left\{\mathbf{x}_{\mathbf{1}}, \ldots, \mathbf{x}_{\mathbf{n}}\right\}$ denotes the set of state vertices, $\mathbf{Y}=\left\{\mathbf{y}_{\mathbf{1}}, \ldots, \mathbf{y}_{\mathbf{p}}\right\}$ denotes the set of output vertices, $\mathbf{Z}=\left\{\mathbf{z}_{\mathbf{1}}, \ldots, \mathbf{z}_{\mathbf{q}}\right\}$ denotes the set of dynamic variable vertices and $\mathcal{E}=E$-edges $\cup A$-edges $\cup C$-edges with, $E$-edges $=\left\{\left(\mathbf{x}_{\mathbf{i}}, \mathbf{z}_{\mathbf{j}}\right) \mid E_{j, i}^{\lambda} \neq 0\right\}, A$-edges $=\left\{\left(\mathbf{z}_{\mathbf{i}}, \mathbf{x}_{\mathbf{j}}\right) \mid A_{i, j}^{\lambda} \neq 0\right\}$, $C$-edges $=\left\{\left(\mathbf{y}_{\mathbf{i}}, \mathbf{x}_{\mathbf{j}}\right) \mid C_{i, j}^{\lambda} \neq 0\right\}$. Here $M_{i, j}^{\lambda}$ is the $(i, j)$ th element of matrix $M^{\lambda}$ and $\left(\mathbf{s}_{\mathbf{1}}, \mathbf{s}_{\mathbf{2}}\right)$ denotes a directed edge from vertex $\mathbf{s}_{\mathbf{1}} \in \mathcal{V}$ to vertex $\mathbf{s}_{\mathbf{2}} \in \mathcal{V}$. Note that to differentiate the state, the output and the dynamic variables from their corresponding vertices, the latter are written in bold font.

The digraph we define above has not exactly the same structure than the one used in (Dion et al., 2003; Reinschke and Wiedemann, 1997; Wiedemann, 1999) because we add vertex subset $\mathbf{Z}$. This set allows us to remove all assumptions on matrix pencil $\left[s E^{\lambda}-A^{\lambda}\right]$ and on the regularity of the system. Readers interested by different representations in graphtheoretic approach can refer to (Murota, 1987; Reinschke, 1988). Hereafter, we illustrate the proposed digraph definition with an example.

Example 2 Consider the structured system defined by:

$E^{\lambda}=\left(\begin{array}{ccccc}\lambda_{1} & \lambda_{2} & 0 & 0 & 0 \\ 0 & \lambda_{3} & \lambda_{4} & 0 & 0 \\ 0 & 0 & \lambda_{5} & 0 & 0\end{array}\right), \quad A^{\lambda}=\left(\begin{array}{ccccc}0 & 0 & 0 & \lambda_{6} & \lambda_{7} \\ 0 & 0 & \lambda_{8} & 0 & \lambda_{9} \\ \lambda_{10} & 0 & 0 & 0 & 0\end{array}\right)$ and $C^{\lambda}=$ $\left(\begin{array}{ccccc}\lambda_{11} & \lambda_{12} & 0 & 0 & 0 \\ 0 & 0 & \lambda_{13} & 0 & 0\end{array}\right)$. To such a model, we associate the digraph in figure 1 .

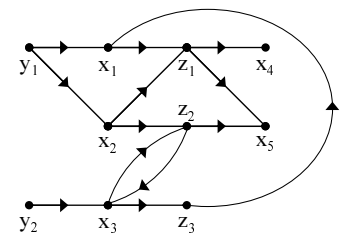

Figure 1. Example 2

We give now some helpful definitions and notations.

- For each edge $e=\left(\mathbf{s}_{\mathbf{0}}, \mathbf{s}_{\mathbf{1}}\right) \in \mathcal{E}, \mathbf{s}_{\mathbf{0}}$ is the begin vertex and $\mathbf{s}_{\mathbf{1}}$ is the end vertex of $e$. Two edges $e_{1}=\left(\mathbf{s}_{\mathbf{1}}, \mathbf{s}_{\mathbf{1}}^{\prime}\right)$ and $e_{2}=\left(\mathbf{s}_{\mathbf{2}}, \mathbf{s}_{\mathbf{2}}^{\prime}\right)$ are $v$-disjoint if $\mathbf{s}_{\mathbf{1}} \neq \mathbf{s}_{\mathbf{2}}$ and $\mathbf{s}_{\mathbf{1}}^{\prime} \neq \mathbf{s}_{\mathbf{2}}^{\prime}$. Note that $e_{1}$ and $e_{2}$ can be $v$-disjoint even if $\mathbf{s}_{\mathbf{1}}^{\prime}=\mathbf{s}_{\mathbf{2}}$ or $\mathbf{s}_{\mathbf{1}}=\mathbf{s}_{\mathbf{2}}^{\prime}$. Some edges are $v$-disjoint if they are mutually $v$-disjoint.

- A subgraph $\mathcal{S}_{\mathcal{G}}=\left(\mathcal{S}_{\mathcal{V}}, \mathcal{S}_{\mathcal{E}}\right)$ of $\mathcal{G}\left(\Sigma_{\Lambda}\right)$ is defined by an edge subset $\mathcal{S}_{\mathcal{E}} \subseteq \mathcal{E}$ and a vertex subset $\mathcal{S}_{\mathcal{V}} \subseteq \mathcal{V}$ such that $\mathcal{S}_{\mathcal{V}}$ is constituted by the begin and the end vertices of all the edges of $\mathcal{S}_{\mathcal{E}} . \mathcal{S}_{\mathcal{G}}$ is said to cover a vertex $\mathbf{s}$ if there exists an edge $e \in \mathcal{S}_{\mathcal{E}}$ such that $\mathbf{s}$ is the end vertex of $e$. Moreover, $\mathcal{S}_{\mathcal{G}}$ is a $v$-disjoint subgraph if all its edges are $v$-disjoint.

- Let $2^{\mathcal{V}}$ denote the power set of set $\mathcal{V}$ composed of all the subsets of $\mathcal{V}$. Function $\varphi$ is defined by:

$\varphi: 2^{\mathcal{V}} \rightarrow 2^{\mathcal{V}}$

$V_{0} \mapsto V_{1}=\varphi\left(V_{0}\right)=\left\{\mathbf{s} \in \mathcal{V} /\left(\mathbf{s}_{\mathbf{0}}, \mathbf{s}\right) \in \mathcal{E}, \quad\right.$ where $\left.\mathbf{s}_{\mathbf{0}} \in V_{0}\right\}$ $\varphi\left(V_{0}\right)$ represents the reachable set from $V_{0}$ using any edge of $\mathcal{E}$ beginning in $V_{0}$. We define also function $\bar{\varphi}$ such that:

$$
\begin{aligned}
\bar{\varphi}: & 2^{\mathcal{V}} \rightarrow 2^{\mathcal{V}} \\
V_{1} & \mapsto \bar{V}_{0}=\bar{\varphi}\left(V_{1}\right)=\left\{\mathbf{s} \in \mathcal{V} /\left(\mathbf{s}, \mathbf{s}_{\mathbf{1}}\right) \in \mathcal{E}, \text { where } \mathbf{s}_{\mathbf{1}} \in V_{1}\right\}
\end{aligned}
$$

\section{Main results}

The observability of descriptor systems has been mainly studied using geometric and algebraic tools (Cobb, 1984; Yip and Sincovec, 1981; Verghese et al., 1981; Özccaldiran et al., 1992; Hou and Müller, 1999a). In this context, the originality of our work, based on graph theory, is to provide observability conditions which have an intuitive interpretation and are very simple to check. Moreover, our approach allows us to characterize also the right-hand observability of any given part of the state. The graphic conditions, given in this paper, are related to the existence of a $v$-disjoint subgraph which covers all the state vertices, knowing that some $A$-edges cannot be used in such subgraph, due to the presence of matrix $E^{\lambda}$. Therefore, the first part of this section is devoted to determinate which edges can be used. These edges are called useful edges.

\subsection{Determination of the useful vertices of $\mathcal{G}\left(\Sigma_{\Lambda}\right)$}

The theorems giving necessary and sufficient conditions to ensure the observability of linear systems in standard form are recalled in (Dion et al., 2003). These theorems cannot be directly applied to descriptor systems mainly because they assume that all the derivatives of the outputs are exploitable to observe the state. This assumption is not always verified in the case of descriptor systems, particularly when the output derivatives cannot be expressed in function of the state and the input. Effectively, recall that the derivative of $H^{\lambda} x$ can be generically expressed in function of the state and the input iff $\operatorname{Im}\left(H^{\lambda^{T}}\right) \subseteq \operatorname{Im}\left(E^{\lambda^{T}}\right)$. In this case, we say that $H^{\lambda} x$ has a useful derivative. We precise hereafter the notion of useful derivative:

Definition 3 Consider $\left(\Sigma_{\Lambda}\right)$, we say that $\sum_{i=0}^{r} \sigma_{i} y^{(i)}$ has generically a useful derivative iff there exist an integer $l$ and matrices $\Gamma^{\lambda}$ and $\Gamma_{u}^{\lambda}$ such that $\sum_{i=0}^{r} \sigma_{i} y^{(i)}=\Gamma^{\lambda} x+$ $\Gamma_{u}^{\lambda}\left(u^{T}, \dot{u}^{T}, \ldots,\left(u^{(l)}\right)^{T}\right)^{T}$, with $\operatorname{Im}\left(\Gamma^{\lambda^{T}}\right) \subseteq \operatorname{Im}\left(E^{\lambda^{T}}\right)$ for almost all the realizations of structured system $\left(\Sigma_{\Lambda}\right)$, or equivalently, if there exist an integer $l$ and matrices $P^{\lambda}$ and $\Gamma_{u}^{\lambda}(t)$ of appropriate dimensions such that $\sum_{i=0}^{r} \sigma_{i} y^{(i)}=$ $P^{\lambda} z+\Gamma_{u}^{\lambda}\left(u^{T}, \dot{u}^{T}, \ldots,\left(u^{(l)}\right)^{T}\right)^{T} \cdot \sum_{i=0}^{r} \sigma_{i} y^{(i+1)}$ is useful to $o b-$ serve the state only when $\sum_{i=0}^{r} \sigma_{i} y^{(i)}$ has generically a useful derivative.

A first step to analyze the r-h.s. observability of descriptor systems is to determine which edges, or equivalently which dynamical vertices correspond to useful derivatives of the output and so can be used to observe the state. In this respect, let us precise the notion of combined vertices which correspond to a linear combination of dynamical variables. 
- We denote by $\mathbf{z}_{\mathbf{i}_{1}, \mathbf{i}_{2}, \ldots, \mathbf{i}_{\mathbf{k}}}$ the vertex associated to any combination of dynamic variables $z_{i_{1}}, z_{i_{2}}, \ldots, z_{i_{k}}$ whatever $i_{1}<i_{2}<\ldots<i_{k}$.

$\mathbf{Z}_{\mathbf{i}_{1}, \mathbf{i}_{2}, \ldots, \mathbf{i}_{\mathbf{k}}}$, which we call combined vertex, can be defined by means of bijective function $\pi$ associating a combined vertex to every nonempty subset of $\mathbf{Z}$ : $\left\{\mathbf{z}_{\mathbf{i}_{1}}, \mathbf{z}_{\mathbf{i}_{2}}, \ldots, \mathbf{z}_{\mathbf{i}_{\mathbf{k}}}\right\} \mapsto \pi\left(\left\{\mathbf{z}_{\mathbf{i}_{1}}, \mathbf{z}_{\mathbf{i}_{2}}, \ldots, \mathbf{z}_{\mathbf{i}_{\mathbf{k}}}\right\}\right) \stackrel{\text { def }}{=} \mathbf{z}_{\mathbf{i}_{1}, \mathbf{i}_{2}, \ldots, \mathbf{i}_{\mathbf{k}} .}$.

We denote by $\bar{\pi}$ the inverse function of $\pi$. The set of combined vertices is noted $\mathbf{Z}_{\mathbf{C}}$. Obviously, $\mathbf{Z}_{\mathbf{C}}$ includes $\mathbf{Z}$.

- A nonempty vertex subset $\mathbf{V}_{\mathbf{C}} \subseteq \mathbf{Z}_{\mathbf{C}}$ is said "structurally minimal" if $\forall \overline{\mathbf{V}} \subseteq \mathbf{V}_{\mathbf{C}}$,

$\operatorname{card}\left(\bigcup_{\mathbf{v} \in \overline{\mathbf{V}}} \bar{\pi}(\mathbf{v})\right)-\operatorname{card}(\overline{\mathbf{V}}) \geq \max _{\mathbf{v} \in \overline{\mathbf{V}}}(\operatorname{card}(\bar{\pi}(\mathbf{v})))-1$.

For example, $\mathbf{V}_{1}=\left\{\mathbf{z}_{1}, \mathbf{z}_{\mathbf{2}, 3}, \mathbf{z}_{2,4}\right\}$ is structurally minimal while $\mathbf{V}_{2}=\left\{\mathbf{z}_{\mathbf{1}, \mathbf{2}, \mathbf{3}}, \mathbf{z}_{\mathbf{2}, \mathbf{3}}, \mathbf{z}_{\mathbf{2}, \mathbf{4}}\right\}$ is not structurally minimal be-

cause for $\overline{\mathbf{V}}=\left\{\mathbf{z}_{\mathbf{1}, \mathbf{2}, \mathbf{3}}, \mathbf{z}_{2, \mathbf{3}}\right\}, \operatorname{card}\left(\bigcup_{\mathbf{v} \in \overline{\mathbf{V}}} \bar{\pi}(\mathbf{v})\right)-\operatorname{card}(\overline{\mathbf{V}})=3-2$ and $\max _{\mathbf{v} \in \overline{\mathbf{V}}}(\operatorname{card}(\bar{\pi}(\mathbf{v})))-1=3-1$.

$$
\mathbf{v} \in \overline{\mathbf{V}}
$$

- To each $\mathbf{V}_{\mathbf{C}} \subseteq \mathbf{Z}_{\mathbf{C}}$, we can associate a vertex subset $\wp\left(\mathbf{V}_{\mathbf{C}}\right)$ constituted by all the elements $\mathbf{s} \in \mathbf{Z}_{\mathbf{C}}$ for which there exists $\mathbf{V}_{\mathbf{0}} \subseteq \mathbf{V}_{\mathbf{C}}$ such that:

$\left\{\begin{array}{l}\operatorname{card}\left(\bigcup_{\mathbf{v} \in \mathbf{V}_{\mathbf{0}}} \bar{\pi}(\mathbf{v})\right)-\operatorname{card}(\bar{\pi}(\mathbf{s}))=\operatorname{card}\left(\mathbf{V}_{\mathbf{0}}\right)-1 \\ \forall \overline{\mathbf{V}}_{\mathbf{0}} \subset \mathbf{V}_{\mathbf{0}} \mid \operatorname{card}\left(\overline{\mathbf{V}}_{\mathbf{0}}\right)=\operatorname{card}\left(\mathbf{V}_{\mathbf{0}}\right)-1, \\ \qquad \bigcup_{\mathbf{v} \in \overline{\mathbf{V}}_{\mathbf{0}}} \bar{\pi}(\mathbf{v}) \cup \bar{\pi}(\mathbf{s})=\bigcup_{\mathbf{v} \in \mathbf{V}_{\mathbf{0}}} \bar{\pi}(\mathbf{v})\end{array}\right.$

For example, for $\mathbf{V}_{\mathbf{C}}=\left\{\mathbf{z}_{\mathbf{1}, \mathbf{2}}, \mathbf{z}_{1,3}, \mathbf{z}_{\mathbf{4}}\right\}$, we have $\wp\left(\mathbf{V}_{\mathbf{C}}\right)=\left\{\mathbf{z}_{1,2}, \mathbf{z}_{1,3}, \mathbf{z}_{2,3}, \mathbf{z}_{\mathbf{4}}\right\}$. Note that $\forall \mathbf{V}_{\mathbf{Z}} \subseteq \mathbf{Z}$ we have $\wp\left(\mathbf{V}_{\mathbf{Z}}\right)=\mathbf{V}_{\mathbf{Z}}$. Obviously, we have $\forall \mathbf{V}_{\mathbf{C}} \subseteq \mathbf{Z}_{\mathbf{C}}, \mathbf{V}_{\mathbf{C}} \subseteq \wp\left(\mathbf{V}_{\mathbf{C}}\right)$. - The definition of $\varphi$ in section 3 is adapted for taking into account $\mathbf{Z}_{\mathbf{C}}$. In the sequel, $\varphi_{e}$ is such that:

$$
\begin{aligned}
\varphi_{e}: 2^{\mathbf{Y} \cup \mathbf{Z}_{\mathbf{C}}} & \rightarrow 2^{\mathbf{X} \cup \mathbf{Z}_{\mathbf{C}}} \\
V_{0} & \mapsto \varphi_{e}\left(V_{0}\right)=V_{1}=\left(\bigcup_{s \in V_{0} \cap \mathbf{Z}_{\mathbf{C}}} \varphi(\bar{\pi}(s))\right) \cup \varphi\left(V_{0} \cap \mathbf{Y}\right)
\end{aligned}
$$

- We also define functions $\theta$ and $\bar{\theta}$ as:

$$
\begin{aligned}
& \theta: 2^{\mathbf{Y} \cup \mathbf{Z}_{\mathbf{C}}} \rightarrow \mathbb{N} \\
& V_{0} \mapsto \theta\left(V_{0}\right)=\text { maximal number of } v \text {-disjoint } \\
& \bar{\theta}: 2^{\mathbf{Z}} \rightarrow \mathbb{N} \quad \text { edges from } V_{0} \text { to } \varphi_{e}\left(V_{0}\right) \\
& V_{0} \mapsto \bar{\theta}\left(V_{0}\right)=\text { maximal number of } v \text {-disjoint } \\
& \text { edges from } \bar{\varphi}\left(V_{0}\right) \text { to } V_{0}
\end{aligned}
$$

knowing that, edge subset $A_{C}$-edges $=$

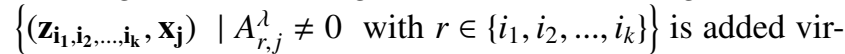
tually to the digraph (there is no need to redraw the digraph associated to the system) to take into account $\mathbf{Z}_{\mathbf{C}}$.

Functions $\theta$ and $\bar{\theta}$ have the following properties:

P1. Let $\tilde{\mathbf{Y}} \subseteq \mathbf{Y}$ and note by $\tilde{y}$ its associated vector. Let $C^{\lambda}$ the matrix such that $\tilde{y}=\tilde{C}^{\lambda} x$, we have $\theta(\tilde{\mathbf{Y}})=g-\operatorname{rank}\left[\tilde{C}^{\lambda}\right]$, where $g$-rank denotes the generic rank (Reinschke, 1988).

P2. Similarly, let $\tilde{\mathbf{Z}} \subseteq \mathbf{Z}$ and note by $\tilde{z}$ its associated vector. Let $E^{\lambda}$ the matrix such that $\tilde{z}=\tilde{E}^{\lambda} x$, we have that $\bar{\theta}(\tilde{\mathbf{Z}})=g-\operatorname{rank}\left[\tilde{E}^{\lambda}\right]$.
In order to graphically analyse the observability of structured descriptor systems, we follow an iterative approach where the $i^{\text {th }}$ stage corresponds to the $i^{\text {th }}$ differentiation order of the output. To observe the state, only useful derivatives of the output can be used. Determining these derivatives amounts to find the elements of $A$-edges, or equivalently the vertices of $\mathbf{Z}_{\mathbf{C}}$, corresponding to the useful derivatives of the output. These edges (resp. vertices) are called useful edges (resp. vertices).

Let us denote $\mathbf{M}_{\mathbf{0}}=\mathbf{Y}$. If $\theta\left(\mathbf{M}_{\mathbf{0}}\right)=n$, then there is no need to resort to the output derivatives to observe the state. Indeed, in this case, generic rank of $C^{\lambda}$ is equal to the state dimension and so the system is observable. Otherwise, a first differentiation of the output is necessary to achieve the observability of the whole state. In this respect, we must find vertices of $z_{i_{1}, i_{2}, \ldots, i_{k}}$ of $\mathbf{Z}_{\mathbf{C}}$ which can express the useful first derivatives of the output i.e. there exists generically a matrix $\Gamma^{\lambda}$ such that $\Gamma^{\lambda} y$ can be expressed using a combination of $z_{i_{1}}, \ldots z_{i_{k}}$. The following lemma allows us to determinate which vertices of $\mathbf{Z}_{\mathbf{C}}$ are useful. Note that only linearly independent equations linking $y$ and its derivatives to the state are useful to achieve the state observability.

Lemma 4 Consider a subset $\Omega_{\mathbf{z}}=\left\{\mathbf{z}_{\mathbf{i}_{1}}, \mathbf{z}_{\mathbf{i}_{2}}, \ldots \mathbf{z}_{\mathbf{i}_{\mathbf{k}}}\right\}$ and its corresponding combined vertex $\mathbf{z}_{\mathbf{i}_{1}, \mathbf{i}_{2}, \ldots, \mathbf{i}_{\mathbf{k}}}$. Assume that

$\nexists \Omega_{\mathrm{z}}^{\prime} \subset \Omega_{\mathrm{z}}$ such that $\pi\left(\Omega_{\mathrm{z}}^{\prime}\right)$ is useful. $\pi\left(\Omega_{\mathrm{z}}\right)$ is useful or equivalently there exist vectors $P^{\lambda} \in \mathbb{R}^{1 \times k}$ and $\Gamma^{\lambda} \in \mathbb{R}^{1 \times p}$ satisfying $P^{\lambda}\left(z_{i_{1}}, \ldots, z_{i_{k}}\right)^{T}=\Gamma^{\lambda} y$ iff $\exists \Omega_{\mathbf{M}} \subseteq \mathbf{M}_{\mathbf{0}}$ such that:

$$
\theta\left(\Omega_{\mathbf{M}}\right)=\operatorname{card}\left(\varphi_{e}\left(\Omega_{\mathbf{M}}\right) \cup \bar{\varphi}\left(\Omega_{\mathbf{z}}\right)\right)-\theta\left(\Omega_{\mathbf{z}}\right)+1
$$

Proof of sufficiency: Assume that condition (2) is satisfied and let us take the following notations: $x_{\Omega}$ is the vector of the state components represented by the elements of vertex set $\varphi_{e}\left(\Omega_{\mathbf{M}}\right) \cup \bar{\varphi}\left(\Omega_{\mathbf{z}}\right), z_{\Omega}$ is the vector of the dynamic variables associated to $\Omega_{\mathbf{z}}$ and $y_{\Omega}$ is the vector of the output components associated to $\Omega_{\mathbf{M}}$.

Using these notations, we can write $y_{\Omega}=C_{\Omega} x_{\Omega}$ and $z_{\Omega}=E_{\Omega} x_{\Omega}$. Relation (2) of lemma 4 implies that generically $\operatorname{dim}\left(\operatorname{Im}\left(C_{\Omega}^{T}\right)\right)+\operatorname{dim}\left(\operatorname{Im}\left(E_{\Omega}^{T}\right)\right)=\operatorname{dim}\left(x_{\Omega}\right)+1$. Then, immediately we can conclude that $\operatorname{dim}\left(\operatorname{Im}\left(E_{\Omega}^{T}\right) \cap \operatorname{Im}\left(C_{\Omega}^{T}\right)\right) \geq 1$. Consequently, generically there exist line vectors $P^{\lambda} \in \mathbb{R}^{1 \times k}$ and $\Gamma_{\Omega}^{\lambda} \in \mathbb{R}^{1 \times p_{\Omega}}$ such that $P^{\lambda} E_{\Omega} x_{\Omega}=\Gamma_{\Omega}^{\lambda} C_{\Omega} x_{\Omega} \Rightarrow \exists \Gamma^{\lambda}$ such that $P^{\lambda} z_{\Omega}=\Gamma^{\lambda} y$. This proves the sufficiency.

Note that $\theta\left(\Omega_{\mathbf{M}}\right) \geq \operatorname{card}\left(\varphi_{e}\left(\Omega_{\mathbf{M}}\right) \cup \bar{\varphi}\left(\Omega_{\mathbf{z}}\right)\right)-\bar{\theta}\left(\Omega_{\mathbf{z}}\right)+1$ is also sufficient to prove the existence of vectors $P^{\lambda} \in \mathbb{R}^{k}$ and $\Gamma^{\lambda} \in \mathbb{R}^{1 \times p}$ such that $P^{\lambda} z_{\Omega}=\Gamma^{\lambda} y$.

Proof of necessity: Assume now that there exist line vectors $P^{\lambda} \in \mathbb{R}^{1 \times k}$ and $\Gamma^{\lambda} \in \mathbb{R}^{p}$ such that $P^{\lambda} z_{\Omega}=\Gamma^{\lambda} y$. Let $y_{\Omega}$ be the vector of components of $y$ which intervenes in $\Gamma^{\lambda} y$ i.e. $\Gamma^{\lambda} y=\Gamma_{\Omega}^{\lambda} y_{\Omega}$ and let $\Omega_{\mathbf{M}}$ be the vertex subset associated to the components of $y_{\Omega}$.

From the construction of the digraph, $P^{\lambda} z_{\Omega}$ is a linear function of $x_{j}$ where $\mathbf{x}_{\mathbf{j}} \in \bar{\varphi}\left(\Omega_{\mathbf{z}}\right)$. To satisfy $P^{\lambda} z_{\Omega}=\Gamma^{\lambda} y=\Gamma_{\Omega}^{\lambda} y_{\Omega}$, the variables corresponding to $\bar{\varphi}\left(\Omega_{\mathrm{z}}\right)$ are necessarily either eliminated in combination $P_{k}^{\lambda} z_{\Omega}$ or can be expressed only in function of $y_{\Omega}$. On the one hand, a combination of $k$ dynamical variables $z_{i}$ can eliminate at most $\bar{\theta}\left(\Omega_{\mathbf{z}}\right)-1$ elements of $\bar{\varphi}\left(\Omega_{\mathbf{z}}\right)$. Let $q=\operatorname{card}\left[\bar{\varphi}\left(\Omega_{\mathbf{z}}\right)\right]$. Thus, at least 
$q-\bar{\theta}\left(\Omega_{\mathbf{z}}\right)+1$ variables corresponding to the vertices included in $\bar{\varphi}\left(\Omega_{\mathrm{z}}\right)$ must be generically expressed in function of $y_{\Omega}$ to satisfy equation $P^{\lambda} z_{\Omega}=\Gamma_{\Omega}^{\lambda} y_{\Omega}$. However, $y_{\Omega}$ is a linear function of the state components associated to $\varphi_{e}\left(\Omega_{\mathbf{M}}\right)$, which are not necessarily included in $\bar{\varphi}\left(\Omega_{\mathbf{z}}\right)$. Thus, the components of $\varphi_{e}\left(\Omega_{\mathbf{M}}\right) \backslash\left(\bar{\varphi}\left(\Omega_{\mathbf{z}}\right) \cap \varphi_{e}\left(\Omega_{\mathbf{M}}\right)\right)$ must be added to the components which must be expressed using $y_{\Omega}$. The number of state variables we can express using $y_{\Omega}$ is equal to $\theta\left(\Omega_{\mathbf{M}}\right)$. Thus, $\Omega_{\mathbf{M}}$ must satisfy $\theta\left(\Omega_{\mathbf{M}}\right) \geq n_{1} \stackrel{\text { def }}{=} \operatorname{card}\left(\varphi_{e}\left(\Omega_{\mathbf{M}}\right) \cup \bar{\varphi}\left(\Omega_{\mathbf{z}}\right)\right)-\bar{\theta}\left(\Omega_{\mathbf{z}}\right)+1$.

Moreover, assume now that there exists $\Omega_{\mathbf{M}} \subseteq \mathbf{M}_{\mathbf{0}}$ such that $\theta\left(\Omega_{\mathbf{M}}\right)=n_{1}^{\prime}>n_{1}$. Then, for all $\Omega_{\mathbf{z}}^{\prime} \subset \Omega_{\mathbf{z}}$ such that $\operatorname{card}\left(\Omega_{\mathrm{z}}^{\prime}\right)=\operatorname{card}\left(\Omega_{\mathrm{z}}\right)-1$, we have $\theta\left(\Omega_{\mathbf{M}}\right) \geq \operatorname{card}\left(\varphi_{e}\left(\Omega_{\mathbf{M}}\right) \cup \bar{\varphi}\left(\Omega_{\mathbf{z}}^{\prime}\right)\right)-\bar{\theta}\left(\Omega_{\mathbf{z}}^{\prime}\right)+1$. According to the sufficiency of lemma 4 proved above, $\pi\left(\Omega_{\mathrm{z}}^{\prime}\right)$ is useful. However, it was assumed that $\nexists \Omega_{\mathrm{z}}^{\prime} \subset \Omega_{\mathrm{z}}$ where $\pi\left(\Omega_{\mathrm{z}}^{\prime}\right)$ useful. So, there cannot exist $\Omega_{\mathbf{M}} \subseteq \mathbf{M}_{\mathbf{0}}$ such that $\theta\left(\Omega_{\mathbf{M}}\right)=n_{1}^{\prime}>n_{1}$. On the one hand, we have proved the existence of $\Omega_{\mathbf{M}} \subseteq \mathbf{M}_{\mathbf{0}}$ such that $\theta\left(\Omega_{\mathbf{M}}\right) \geq n_{1}$ and, on the other hand, under the assumption of Lemma 4 , we have established that there does not exist $\Omega_{\mathbf{M}} \subseteq \mathbf{M}_{\mathbf{0}}$ such that $\theta\left(\Omega_{\mathbf{M}}\right)>n_{1}$. Consequently, $\exists \Omega_{\mathbf{M}} \subseteq \mathbf{M}_{\mathbf{0}}$ such that $\theta\left(\Omega_{\mathbf{M}}\right)=n_{1}=\operatorname{card}\left(\varphi_{e}\left(\Omega_{\mathbf{M}}\right) \cup \bar{\varphi}\left(\Omega_{\mathbf{z}}\right)\right)-\bar{\theta}\left(\Omega_{\mathbf{z}}\right)+1$.

We present hereafter the algorithm used to determine a vertex subset noted $\mathbf{Z}^{*}$ representing all the useful vertices:

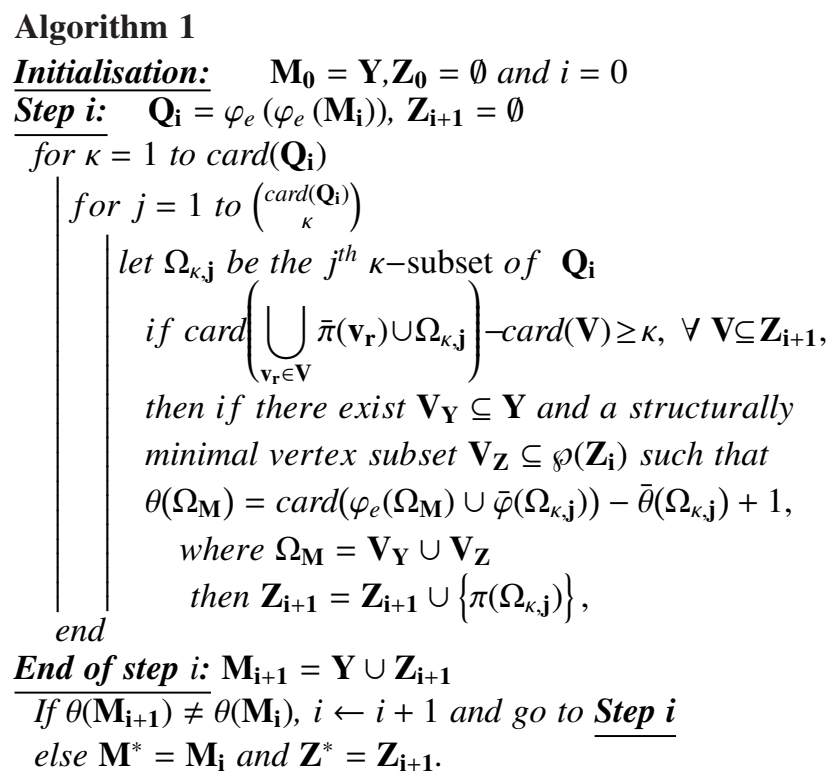

$\mathbf{M}_{\mathbf{0}}=\mathbf{Y}$ represents the output derivatives of order 0 . Lemma 4 states that $\mathbf{Z}_{\mathbf{1}}$ represents all vertices of $\mathbf{Z}_{\mathbf{C}}$ used to express the first derivative of the output in function of $x$. Moreover, $\mathbf{M}_{\mathbf{1}}=\mathbf{M}_{\mathbf{0}} \cup \mathbf{Z}_{\mathbf{1}}$ represents the outputs and their first useful derivatives. This set is sufficient to generically achieve the r-h.s. observability of the system iff $\theta\left(\mathbf{M}_{\mathbf{1}}\right)=$ $\operatorname{card}(\mathbf{X})=n$. Otherwise, the outputs must be differentiated once again. From definition of $\wp(\cdot)$, all the elements $\mathbf{Z}_{\mathbf{i}_{1}, \ldots, \mathbf{i}_{\mathbf{k}}} \in \wp\left(\mathbf{Z}_{\mathbf{1}}\right)$ are such that there exist vectors $P^{\lambda} \in \mathbb{R}^{k}$ and $\Gamma^{\lambda} \in \mathbb{R}^{p}$ satisfying $P^{\lambda}\left(z_{i_{1}}, \ldots, z_{i_{k}}\right)^{T}=\Gamma^{\lambda} y$. Thus, using a similar reasoning to the one of the proof of lemma 4 , and assuming that $\nexists \Omega_{\mathbf{z}}^{\prime} \subset \Omega_{\mathbf{z}}$ such that $\pi\left(\Omega_{\mathbf{z}}^{\prime}\right)$ is useful, we can prove that $\pi\left(\Omega_{\mathbf{z}}\right)$ is useful or equivalently that there exist line vectors $P^{\lambda} \in \mathbb{R}^{1 \times k}$ and $\Gamma_{0}^{\lambda} \in \mathbb{R}^{1 \times p}, \Gamma_{1}^{\lambda} \in \mathbb{R}^{1 \times p}, \Gamma_{u}^{\lambda} \in \mathbb{R}^{1 \times m}$ satisfying $P^{\lambda}\left(z_{i_{1}}, \ldots, z_{i_{k}}\right)^{T}=\Gamma_{0}^{\lambda} y+\Gamma_{1}^{\lambda} \dot{y}+\Gamma_{u} u$ iff there exist $\mathbf{V}_{\mathbf{Y}} \subseteq \mathbf{Y}$ and a structurally minimal vertex subset $\mathbf{V}_{\mathbf{Z}} \subseteq \wp\left(\mathbf{Z}_{\mathbf{1}}\right)$ such that $\theta\left(\Omega_{\mathbf{M}}\right)=\operatorname{card}\left(\varphi_{e}\left(\Omega_{\mathbf{M}}\right) \cup \bar{\varphi}\left(\Omega_{\kappa, \mathbf{j}}\right)\right)-\bar{\theta}\left(\Omega_{\kappa, \mathbf{j}}\right)+1$ where $\Omega_{\mathbf{M}}=\mathbf{V}_{\mathbf{Y}} \cup \mathbf{V}_{\mathbf{Z}}$. Therefore, we deduce that $\mathbf{Z}_{\mathbf{2}}$ represents all vertices of $\mathbf{Z}_{\mathbf{C}}$ used to express the first derivatives of the variables associated to $\mathbf{M}_{\mathbf{1}}$ and so the first and second derivatives of the output in function of $x$. Extending this reasoning, $\mathbf{Z}_{\mathbf{i}+\mathbf{1}}$ represents all vertices of $\mathbf{Z}_{\mathbf{C}}$ used to express the derivatives of order less or equal to $i+1$ of the output in function of $x$. When $\theta\left(\mathbf{M}_{\mathbf{i}+\mathbf{1}}\right)=\theta\left(\mathbf{M}_{\mathbf{i}}\right)$, the $(i+1)^{\text {th }}$ output derivative does not give any new information for the state observation. We can conclude that $\mathbf{Z}^{*}$ represents all vertices of $\mathbf{Z}_{\mathbf{C}}$ used to express the derivatives of the output in function of $x$. Note that, obviously, if the system is r-h.s. observable then $\mathbf{Z}^{*}=\mathbf{Z}$.

\subsection{Observation of the whole state}

The previous issue suggests the following proposition which gives necessary and sufficient conditions to the generic r-h.s. observability of $\left(\Sigma_{\Lambda}\right)$ :

Proposition 5 Consider structured system $\left(\Sigma_{\Lambda}\right)$ represented by digraph $\mathcal{G}\left(\Sigma_{\Lambda}\right)$. ( $\left(\Sigma_{\Lambda}\right)$ is generically r-h.s. observable iff a. $\mathbf{Z}^{*}=\mathbf{Z}$,

b. in graph $\mathcal{G}\left(\Sigma_{\Lambda}\right)$ there exists a v-disjoint subgraph $\mathcal{S}_{\mathcal{G}}$ which covers $\mathbf{X}$.

\subsection{Functional observation case}

The last result we present hereafter concerns the rh.s. observability of only a given part of the state $w=\left(x_{j_{1}}, x_{j_{2}}, \ldots, x_{j_{n^{\prime}}}\right)^{T}$. This problem is important in many applications where only a partial knowledge of the state is required. Note that the matrix pencil approach used to characterize the r-h.s. observability does not allow us to handle easily this problem. Let $\mathbf{w}=\left\{\mathbf{x}_{\mathbf{j}_{1}}, \mathbf{x}_{\mathbf{j}_{2}}, \ldots, \mathbf{x}_{\mathbf{j}_{\mathbf{n}^{\prime}}}\right\}$ denote the vertex subset associated to $w$. As previously, we use algorithm 1 to determine the useful vertices of $\mathbf{Z}_{\mathbf{C}}$. We can suggest directly the following proposition:

Proposition 6 For system $\left(\Sigma_{\Lambda}\right)$ represented by $\mathcal{G}\left(\Sigma_{\Lambda}\right)$, state part $w$ is generically observable iff there exist $\mathbf{V}_{\mathbf{Y}} \subseteq \mathbf{Y}$ and a structurally minimal vertex subset $\mathbf{V}_{\mathbf{Z}} \subseteq \wp\left(\mathbf{Z}^{*}\right)$ such that: $\left\{\begin{array}{l}\mathbf{w} \subseteq \varphi_{e}\left(\mathbf{V}_{\mathbf{Y}} \cup \mathbf{V}_{\mathbf{Z}}\right) \\ \theta\left(\mathbf{V}_{\mathbf{Y}} \cup \mathbf{V}_{\mathbf{Z}}\right)=\operatorname{card}\left(\varphi_{e}\left(\mathbf{V}_{\mathbf{y}} \cup \mathbf{V}_{\mathbf{Z}}\right)\right)\end{array}\right.$

According to Proposition 6, a graphic condition to check the observability of $w$ can be enounced:

Proposition 7 Consider structured system $\left(\Sigma_{\Lambda}\right)$ represented by graph $\mathcal{G}\left(\Sigma_{\Lambda}\right) . w=\left(x_{j_{1}}, \ldots, x_{j_{n^{\prime}}}\right)^{T}$ is generically $r$ h.s. observable iff there exists a $v$-disjoint subgraph $\mathcal{S}_{\mathcal{G}}=$ $\left(\mathcal{S}_{\mathcal{E}}, \mathcal{S}_{\mathcal{V}}\right)$, which covers the vertices of $\mathbf{w}$ and such that: a. no edges of $\mathcal{S}_{\mathcal{G}}$ begin with an element of $\mathbf{Z} \backslash\left(\bigcup_{\mathbf{s} \in \mathbf{Z}^{*}} \bar{\pi}(\mathbf{s})\right)$, b. for each vertex $\mathbf{s} \in \mathbf{Z}^{*}$, only one A-edge beginning with one element of $\bar{\pi}(\mathbf{s})$ can be used in $\mathcal{S}_{\mathcal{G}}$. In the case where such edge is used in $\mathcal{S}_{\mathcal{G}}$, the latter must cover $\varphi_{e}(\{\mathbf{s}\})$, c. all the edges beginning with $\mathbf{s} \in \mathbf{Y}$ can be used in $\mathcal{S}_{\mathcal{G}}$. 
In the case where such edge is used in $\mathcal{S}_{\mathcal{G}}$, the latter must $\operatorname{cover} \varphi_{e}(\{\mathbf{s}\})$.

\section{Example}

For the system of example 2, Algorithm 1 gives $\mathbf{Z}^{*}=\mathbf{Z}$ and in figure 2, we represent a $v$-disjoint subgraph which covers all the state vertices. Thus, this system is generically r-h.s. observable.

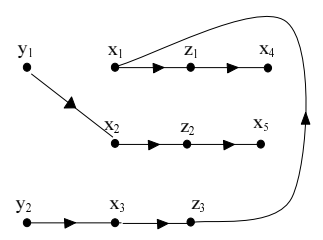

Figure 2. $v$-disjoint subgraph covering $\mathbf{X}$ for example 2

Consider the structured system represented in figure $3 \mathrm{a}$.

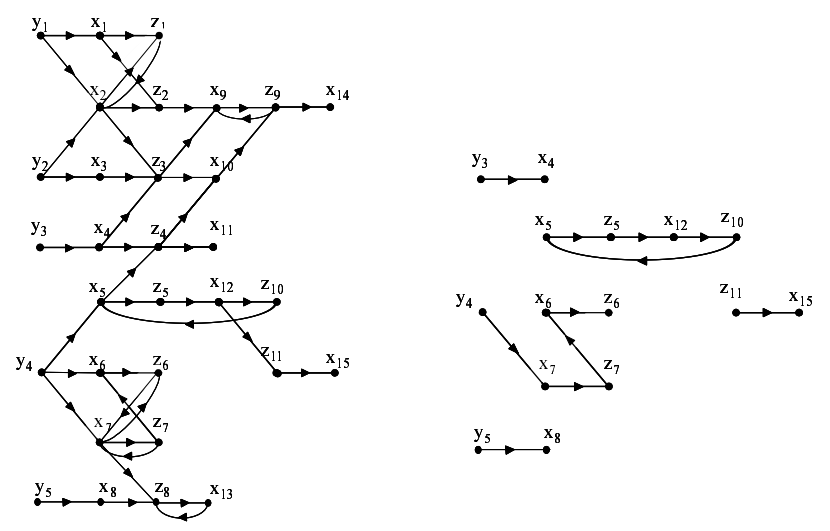

Figure 3. Illustrative example

Using Algorithm 1 , we compute $\mathbf{Z}^{*}=$ $\left\{\mathbf{z}_{1,2}, \mathbf{z}_{2,3}, \mathbf{z}_{1,9}, \mathbf{z}_{\mathbf{4}}, \mathbf{z}_{\mathbf{5}}, \mathbf{z}_{\mathbf{6}}, \mathbf{z}_{7}, \mathbf{z}_{10}, \mathbf{z}_{11}\right\}$. Since $\mathbf{Z}^{*} \neq \mathbf{Z}$, the whole state is not generically observable. However, it is possible search the maximal observable part of the state. The observability subgraph covering the maximal number of state vertices and satisfying conditions of proposition 7 is presented in figure $3 \mathrm{~b}$. Thus, only $w=\left(x_{4}, x_{5}, x_{6}, x_{7}, x_{8}, x_{12}, x_{15}\right)^{T}$ is generically r-h.s. observable.

\section{Conclusion}

In this paper, e a new analysis tool to check the r-h.s. observability of the whole or any given part of structured descriptor systems is proposed. The studied system can be underdetermined, overdetermined or square and obviously non-regular. Using a new graph representation of this kind of systems, necessary and sufficient conditions for the r-h.s. observability are expressed in simple graphic terms. These intrinsic conditions, which do not depend on the parameter values of the system, need few information about the system and so allow us to handle systems with uncertain parameters. Moreover, these conditions are very easy to check by means of well-known combinatorial techniques and simply by hand for small systems. From a computational point of view, note that our approach is particularly suited for large-scale systems and is free from numerical difficulties. Indeed, the proposed algorithm and conditions can be easily implemented because they require simple computations on integers and are based on finding $v$-disjoint subgraphs in graphs. Furthermore, the graph-theoretic approach makes easy the visualization of the system structure. This may be very helpful for the optimisation of sensor placement or for adding sensors to make r-h.s. observable some particular part of the state.

\section{References}

Aplevich, J. D. (1991). Implicit Linear Systems. Springer. New York.

Chu, D., \& Mehrmann, V. (1999). Disturbance decoupled observer design for descriptor systems. System $\mathcal{E}$ Control Letters, 38(1), 37-48.

Cobb, J. D. (1984). Controllability, observability, and duality in singular systems. IEEE Transactions on Automatic Control, AC-29(12), 10761082.

Dai, L. (1989). Singular Control Systems. Springer-Verlag. Berlin.

Darouach, M., Zasadzinski, M., \& Hayar, M. (1996). Reduced order observer design for descriptor systems with unknown inputs. IEEE Transactions on Automatic Control, 41(7), 1068-1072.

Dion, J-M., Commault, C., \& Van der Woude, J. (2003). Generic properties and control of linear structured systems: A survey. Automatica, 39(7), 1125-1144.

Geerts, T. (1993). Solvability conditions, consistency and weak consistency for linear differential-algebraic conditions and timeinvariant singular systems: The general case. Linear Algebra Applications, 181, 111-130.

Hou, M., \& Müller, P. C. (1999a). Causal observability of descriptor systems. IEEE Transactions on Automatic Control, 44(1), 158-163.

Hou, M., \& Müller, P. C. (1999b). Observer design for descriptor systems. IEEE Transactions on Automatic Control, 44(1), 164-169.

Hou, M., \& Patton, R. J. (1998). Input observability and input reconstruction. Automatica, 34(6), 789-794.

Lewis, F. L. (1992). A tutorial on the geometric analysis of linear timeinvariant implicit systems. Automatica, 28(1), 119-137.

Müller, P. C. (2000). Descriptor systems: Pros and cons of system modelling by differential-algebraic equations. Mathematics and Computers in Simulation, 53(4-6), 273-279.

Müller, P. C., \& Hou, M. (1993). On the observer design for descriptor systems. IEEE Transactions on Automatic Control, 38(11), 16661671.

Murota, K. (1987). System Analysis by Graphs and Matroids. SpringerVerlag. New York, U.S.A.

Nikoukah, R., Campbell, S. L., \& Delebecque, F. (1998). Observer design for general linear time-invariant systems. Automatica, 34(5), 575583.

Özccaldiran, K., Fountain, D. W., \& Lewis, F. L. (1992). Some generalized notions of observability. IEEE Transactions on Automatic Control, 37(6), 856-860.

Reinschke, K. J. (1988). Multivariable Control. A Graph Theoretic Approach.. Springer-Verlag. New York, U.S.A.

Reinschke, K. J., \& Wiedemann, G. (1997). Digraph characterization of structural controllability for linear descriptor systems. Linear Algebra and its Applications, 266, 199-217.

Verghese, G. C., Levy, B. C., \& Kailath, T. (1981). A generalized statespace for singular systems. IEEE Transactions on Automatic Control, AC-26(4), 811-831.

Wiedemann, G. (1999). Strukturelle Zugänge Zur Analyse und Synthese Linearer Regelungssysteme in Deskriptorform. shaker verlag ed.. Shaker Verlag. Aaachen, Germany.

Yip, E. L., \& Sincovec, R. F. (1981). Solvability, controllability and observability of continuous descriptor systems. IEEE Transactions on Automatic Control, AC-26(3), 702-707. 